\title{
Grain Subdivision Mechanism Related to Partial Disclinations in Severe Plastic Deformation: A Molecular Dynamics Study
}

\author{
Tomotsugu Shimokawa ${ }^{1, *}$, Tomoaki Yamashita ${ }^{2}$, Tomoaki Niiyama ${ }^{3}$ and Nobuhiro Tsuji ${ }^{4,5}$ \\ ${ }^{1}$ Faculty of Mechanical Engineering, Kanazawa University, Kanazawa 920-1192, Japan \\ ${ }^{2}$ Division of Mechanical Science and Engineering, Kanazawa University, Kanazawa 920-1192, Japan \\ ${ }^{3}$ College of Science and Engineering, Kanazawa University, Kanazawa 902-1192, Japan \\ ${ }^{4}$ Department of Materials Science and Engineering, Kyoto University, Kyoto 606-8501, Japan \\ ${ }^{5}$ Elements Strategy Initiative for Structural Materials (ESISM), Kyoto University, Kyoto 606-8501, Japan
}

Severe plastic deformation (SPD) processes can produce the bulk ultrafine-grained metals with grain sizes of less than $1 \mu$ m. However, the mechanism of grain refinement during SPD is not completely understood. In this study, we perform molecular dynamics simulations of a SPD process like equal-channel angular pressing and demonstrate grain refinement phenomena during the SPD simulations. We propose a new mechanism of grain subdivision related to the mobility of partial disclinations formed in strain-gradient regions during SPD. [doi:10.2320/matertrans.MH201518]

(Received June 15, 2016; Accepted July 4, 2016; Published August 25, 2016)

Keywords: grain refinement, grain subdivision, severe plastic deformation, geometrically necessary boundary, disclination, molecular dynamics

\section{Introduction}

Grain refinement is frequently used to improve the mechanical properties (e.g., strength and toughness) of metals and alloys. For example, grain refinement has been performed in aluminum alloys using recrystallization phenomena during annealing after plastic processing. This procedure reduces the average grain size of the alloy to approximately $10 \mu \mathrm{m}$. Conversely, ultrafine-grained (UFG) structures with grain sizes of less than $1 \mu \mathrm{m}$ can be achieved by imposing extremely large plastic strains on metallic materials during plastic processing; this process is known as severe plastic deformation (SPD). The UFG metals produced by SPD show excellent mechanical properties and have thus attracted the attention of researchers globally ${ }^{1,2)}$. However, the mechanism of grain refinement by SPD is not fully understood. Unlike during grain refinement by recrystallization, UFG structures can be observed immediately after deformation processing at low temperatures in the SPD process; that is, the produced UFG structure is essentially a deformation microstructure formed by deformation.

Based on detailed observations of the microstructural changes after each pass of the SPD process, the concept of grain subdivision has been proposed to explain the grain refinement by $\mathrm{SPD}^{3)}$. The fundamental concept of grain subdivision is that an original grain domain is finely divided by rearranging a large number of dislocations introduced by SPD into energetically stable structures. One of these stable structures is a geometrically necessary boundary (GNB), which can release the elastic strain energy caused by the continuous change in crystal misorientation induced by the difference in slip pattern compared to the original grain. The density of GNBs increases with increasing total plastic strain during the SPD process, while the size of domains subdivided by GNBs decreases, leading to ultrafine (grain) structures ${ }^{4)}$.

*Corresponding author, E-mail: simokawa@se.kanazawa-u.ac.jp
According to the concept of grain subdivision, the formation of GNBs is a necessary condition for grain refinement via SPD.

How are GNBs formed during the SPD process? To make the formation of GNBs easier to understand, we only consider the in-plain components of GNB orientations. As in the simple case of small-angle tilt grain boundaries ${ }^{5)}$, the structure of a GNB with a small misorientation angle can be expressed by an array of edge dislocations with the same sign of the Burgers vector ${ }^{6}$. Groups of dislocations with the same sign of the Burgers vector appear in strain-gradient regions; these dislocations can be known as geometrically necessary dislocations $(\mathrm{GNDs})^{7)}$. A large number of GNDs are required to form GNBs and increase the misorientation of the GNBs. As a result, it is necessary to introduce strain gradients into specimens during SPD to form GNDs.

In fact, most SPD processes produce macroscopic strain-gradient regions in specimens. In principle, the high-pressure torsion (HPT) process introduces a strain gradient outward from the center of the specimen $^{8)}$. In equal-channel angular pressing (ECAP) ${ }^{9,10)}$ and accumulative roll-bonding $(\mathrm{ARB})^{11)}$, the shapes of the specimens are changed by introducing large plastic strains when they pass through the bending point of special dies or the rolls; in other words, macroscopic strain gradients are introduced at the border between a deformed region and an undeformed region via the constraint of deformation tools (e.g., dies and rolls). A strain gradient is also introduced microscopically due to the constraint of deformation by the microstructures of the material (e.g., grain/interphase boundaries).

The above facts suggest that strain gradients play a key role in grain subdivision. Introducing strain gradients via SPD causes grain subdivision through the formation of GNBs by a large number of GNDs generated by the strain gradients. This scenario is difficult to be verified by experimental observations because of the difficulty of direct observations of structural changes in bulky metallic specimens during SPD pro- 
cesses. However, atomic simulations can allow us to directly observe the structural changes at atomic scale during a deformation process with strain gradients.

In this study, we simulate SPD using molecular dynamics and demonstrate grain subdivision phenomena related to strain gradients. We then propose a grain subdivision mechanism governed by the mobility of partial disclinations ${ }^{12)}$ formed around strain-gradient regions.

\section{Analysis Model and Conditions}

First, we discuss the SPD process simulated in this study. Figure 1 shows the analysis model of the SPD process. The dark-gray outer frame is a displacement-controlled (DC) layer consisting of atoms with a crystalline structure. The detailed atomic configurations are given in the next paragraph. The DC layer imposes local shear strain on the specimen by controlling the $x$-directional atomic displacement in the DC layer, as shown in Fig. 1(b). The other directional atomic displacements in the DC layer are fixed. Therefore, the DC layer plays the role of the dies in an ECAP process. The $x$-directional atomic displacement in the DC layer is expressed by a sine wave with an amplitude of $A / 2$ and a wavelength of $\lambda / 2$. We simplify the local shear strain due to the DC layer as the average shear strain $\gamma$ calculated by $A / \lambda$. The local shearstrain zone propagates along the analysis model from the lower part to the upper part with velocity $v$. Strain gradients appear in the analysis model at the boundary area between a deformed region and an undeformed region; hence, strain-gradient regions also propagate during the SPD simulations. The plane-strain condition is adopted to simplify the microstructural changes during the SPD simulations (i.e., the $y$-directional displacements of all atoms are fixed). Consequently, the specimen shape and volume after the SPD simulations remain unchanged from the undeformed ones. Our SPD process is similar to multi-pass ECAP ${ }^{13)}$ and ECAP in parallel channels ${ }^{14)}$. Because the actual SPD processes impose a high hydrostatic pressure on specimens, we impose a homogeneous compressive strain of $5 \%$ in the $x$-direction on the specimen in advance and adopt the atomic positions in the DC layer as the initial state before the SPD simulations.

Next, we explain the virtual crystalline material simulated herein. The specimen adopts a hexagonal close-packed structure. Two (0001) atomic planes are arranged along the $y$-di-

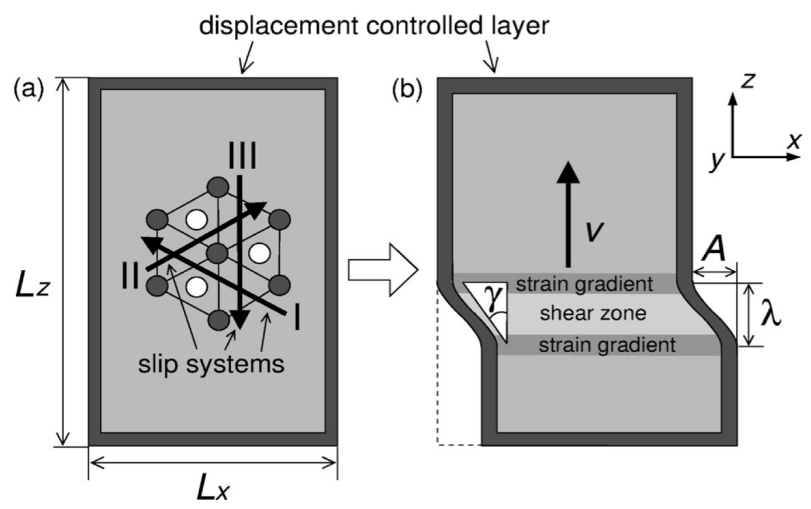

Fig. 1 Schematic of the analysis model of SPD process with local shear strain $\gamma$. rection, and periodic boundary conditions are applied in this direction; hence, only three equivalent prismatic slip systems, the dislocation-line directions of which are parallel to the $y$-direction, can be activated but other slip systems including twin deformation cannot be activated due to geometrical constraints. One of the prismatic slip planes is parallel to the $z$-direction. To facilitate dislocation generation from an interface between the specimen and the DC layer during the SPD simulations, the interface structure is set as an incoherent boundary by arranging atoms at body-centered cubic lattice points in the DC region. The crystal orientations in the DC layer are set as (100), (010), and (001) in the $x$-, $y$-, and $z$-directions, respectively. The embedded atom method for copper proposed by Mishin et al. is used for the interatomic potential ${ }^{15)}$. Although the most stable phase of the EAM potential is face-centered cubic, the phase transformation from hexagonal close-packed to face-centered cubic cannot occur because of the boundary and plane-strain conditions.

The lattice constant $a$ is $0.2556 \mathrm{~nm}$ and the axial ratio for an ideal hexagonal close-packed structure $(c / a=1.633)$ is adopted. The dimensions $L_{x}, L_{y}$, and $L_{z}$ in the analysis model are set at approximately 40,0.42, and $100 \mathrm{~nm}$, respectively. The width of the DC layer is about $2.8 \mathrm{~nm}$. Local shear strain $\gamma$ changes from 0.2 to 1.2 by changing the value of $A$ and fixing $\lambda$ at $20 \mathrm{~nm}$. The propagation speed $v$ of the local shear-strain zone is $500 \mathrm{~m} / \mathrm{s}$, and the temperature is kept at $300 \mathrm{~K}$ using the velocity scaling method ${ }^{16)}$.

\section{Results and Discussion}

\subsection{Grain subdivision by severe plastic deformation}

Figure 2 shows the snapshots from the SPD simulation with $\gamma=0.7$. Atomic colors other than black represent the rotation angle from the initial state, and the black-colored atoms represent defect structures defined using common neighbor analysis ${ }^{17)}$. The atomic colors change discontinuously in the strain-gradient regions, indicating the appearance of crystal misorientations in localized regions. The regions with crystal misorientations propagate along the specimen during the SPD process, and new grains with distinct crystal misorientations finally form after the SPD process, as shown in Fig. 2(g).

Figures 3(a)-3(d) show the distributions of crystal misorientation after SPD with $\gamma=0.2,0.5,0.7$, and 1.0, respectively. For $\gamma=0.2$ and 0.5 , no crystal misorientation appears. On the other hand, for $\gamma=0.7$ and 1.0, new grains are formed. Figure 3(e) shows the detailed atomic configuration in the region with crystal misorientation indicated by the broken box in Fig. 3(c). The defect atoms (colored in black) are classified into two defect types: (1) grain boundaries with misorientation angles of 26 degrees; and (2) line defects such as edge dislocations and vacancy arrays without misorientation angles. The results demonstrate that new deformation microstructures with crystal misorientations can be formed by SPD process with large $\gamma$; we regard this phenomenon as grain subdivision.

Figure 4 shows the proportions of defect atoms after SPD with various values of $\gamma$. Defect atoms are classified into two types, as shown in Fig. 3(e). The classification of defect atoms that compose grain boundaries or line defects is done by 

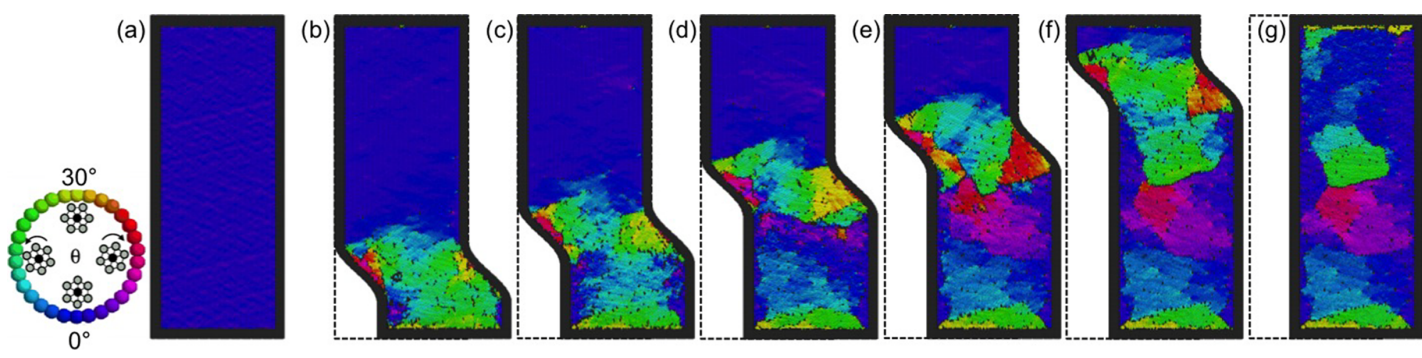

Fig. 2 Grain subdivision caused by SPD process with the propagation of the local shear strain $\gamma=0.7$.
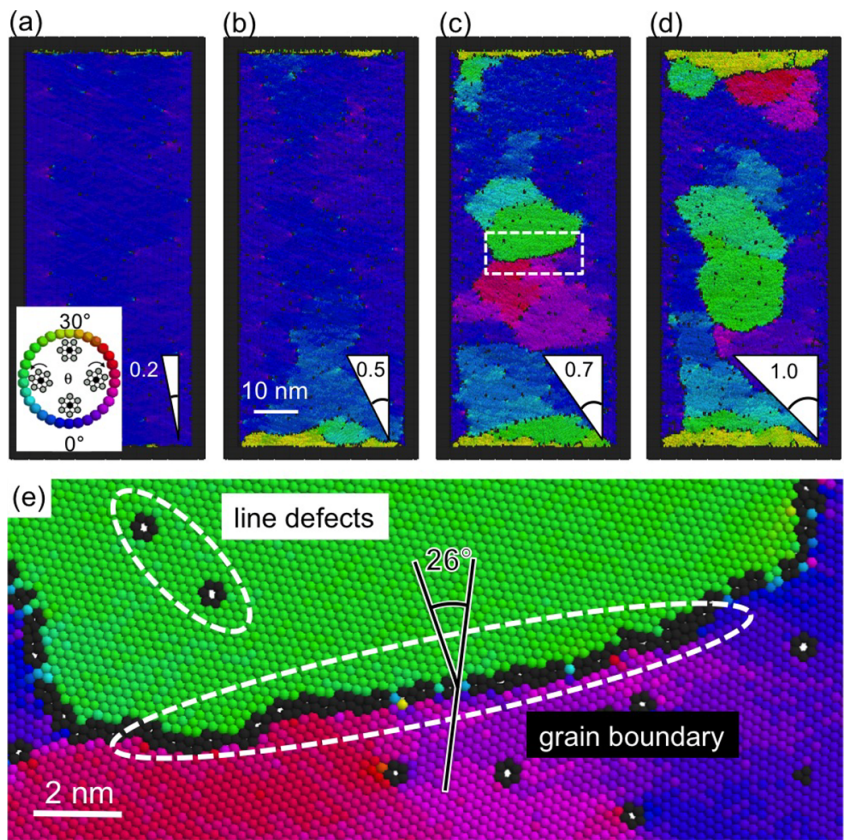

Fig. 3 Cristal orientation maps after SPD processes of (a) $\gamma=0.2$, (b) $\gamma=$ 0.5 , (c) $\gamma=0.7$ and (d) $\gamma=1.0$. (e) Atomic configurations around the grain boundary surrounded by a white broken box in (c)

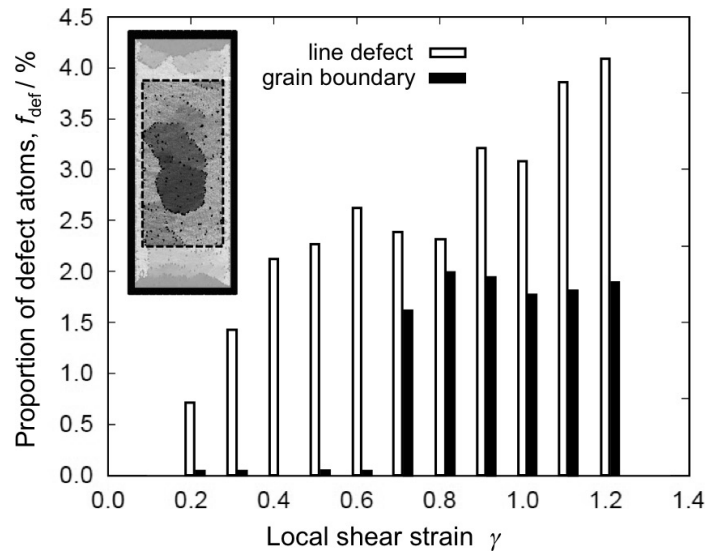

Fig. 4 Local shear strain dependence of the proportion of defect atoms after SPD processes.

considering crystal misorientation angle around the defects atoms: if the crystal misorientation angle is larger than approximately 10 degrees, the defect atoms are classified as ones that compose grain boundaries. We measure the proportion of defect atoms in the central region of the specimens, as shown in the inset of Fig. 4. The proportion of defect atoms
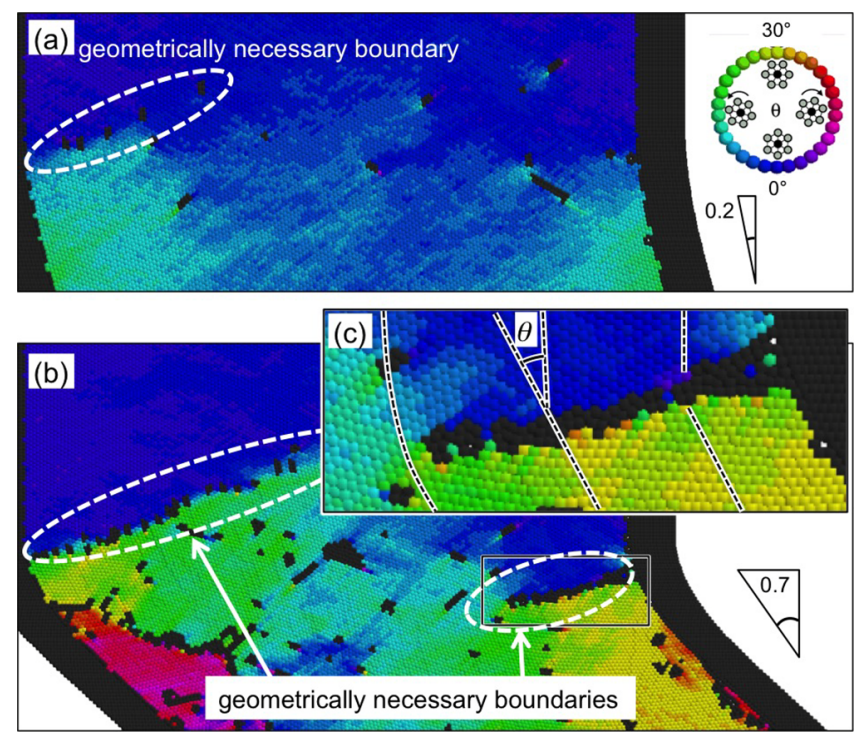

Fig. 5 Distributions of lattice defects around strain-gradient regions during SPD processes. (a) $\gamma=0.2$. (b) $\gamma=0.7$. (c) Enlarged picture of the box region in (b).

that compose line defects increases with $\gamma$. Most line defects are vacancy arrays along the $y$-direction, as shown in Fig. 3(e). The vacancy arrays are formed by the annihilation of parallel dislocations with opposite Burgers vectors; these results imply that the number of dislocations introduced by local shear deformation increases with $\gamma$. On the other hand, the proportion of defect atoms that compose grain boundaries is almost zero when $\gamma<0.6$ and is a constant, non-zero value at $\gamma>0.7$. This clearly demonstrates that a critical local shear strain $\gamma_{\mathrm{c}}$ is necessary to realize grain subdivision during SPD. The grain subdivision mechanism is discussed in detail in the following subsections.

\subsection{Partial disclinations induced by strain gradient}

Figure 5 shows snapshots of defect distributions around strain gradients during the SPD simulations. In the case of $\gamma=$ 0.2 [Fig. 5(a)], line defects arrange at a certain interval around the strain-gradient region on the left side of the specimen, and crystal misorientation appears in that region. This trend is more pronounced in the case of $\gamma=0.7$ [Fig. 5(b)] than in the case of $\gamma=0.2$; line defects on the left side of the specimen arrange at a smaller interval and generate larger crystal misorientation for $\gamma=0.7$ compared to $\gamma=0.2$. On the right side of the specimen with $\gamma=0.7$, a plane defect with large crystal misorientation around the strain-gradient region is observed. 

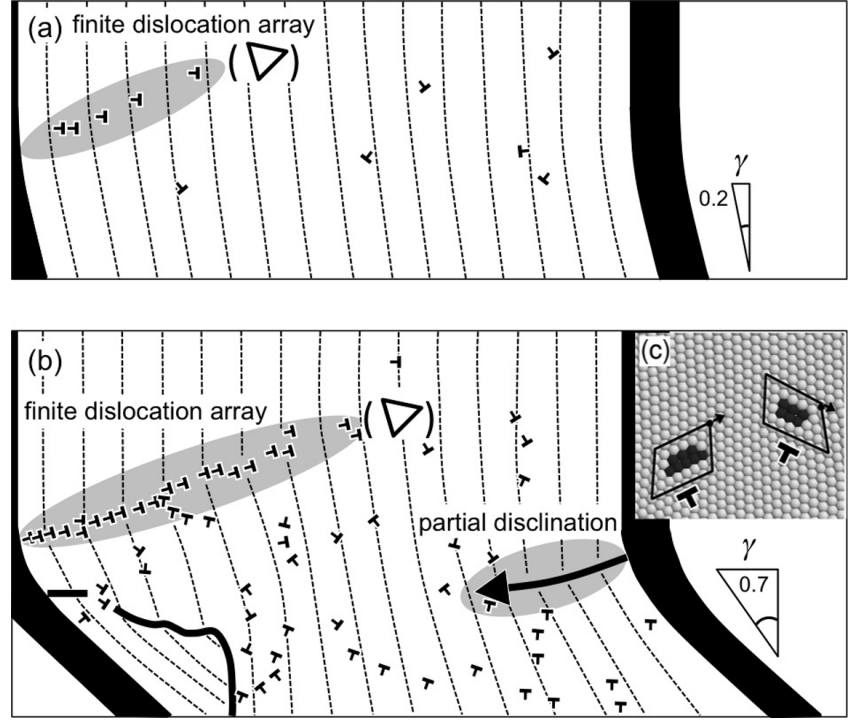

Fig. 6 Partial disclinations accompanied by finite dislocation arrays and a plane defect. The structures of partial-disclination boundaries change according to local shear strain $\gamma$. The filled and unfilled triangle denoting the positive and the negative partial, respectively. (c) Burgers circuits to determine defect structure types.

Since all the above boundaries have crystal misorientation, these boundaries can be regarded as GNBs.

Figure 6 shows the structural analysis results of the lattice defects shown in Fig. 5. By drawing Burgers circuits around all the defects, as shown in the inset of Fig. 6(c), we identify the defect types. The broken lines in Fig. 6 represent the distributions of slip planes in slip system III (Fig. 1). The structural analysis shows that the line defects constituting the GNBs in the left strain-gradient regions of the $\gamma=0.2$ and 0.7 specimens are edge dislocations (Fig. 6). In other words, the GNBs in the left regions are formed by semi-finite edge dislocation arrays with the same sign of the Burgers vector. However, the GNB structure on the right side of the specimen with $\gamma=0.7$ is no longer expressed by a dislocation array; the enlarged image of the plain defect shown in Fig. 5(c) clearly shows that the plain defect is a grain boundary with misorientation angle $\theta=24^{\circ}$.

Interestingly, one termination of each GNB exists inside of the specimen; therefore, the GNBs can be regarded as partial disclinations with Frank vector $\omega^{12)}$. The magnitude of $\omega$ represents the strength of the disclination. In an experiment, partial disclination dipoles are observed in body-centered-cubic iron that had undergone SPD ${ }^{18}$, and the concept of disclination is useful to express the unique mechanical properties and fracture phenomena of UFG and nanocrystalline materials $^{19-21)}$. A partial disclination is accompanied by a terminated boundary, so we call the boundary as the partial-disclination boundary in this study. Generally, the misorientation angle $\theta$ of the partial-disclination boundary is equivalent to the magnitude of $\omega$ (Fig. 6(b)). Consequently, the structure of the partial-disclination boundary changes according to the magnitude of $\omega$.

Figure 7 shows the average misorientation angles $\theta$ of partial-disclination boundaries during the SPD for different values of local shear strain $\gamma$. The average misorientation angles for each $\gamma$ is evaluated from several snapshots during the SPD

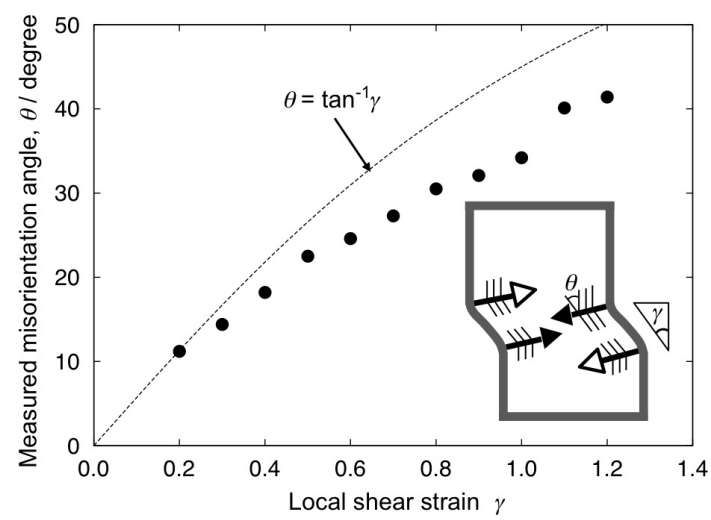

Fig. 7 Relationship between the measured misorientation angle $\theta$ of partial-disclination boundaries and the local shear strain $\gamma$. $\theta$ corresponds to the Frank vector standing for the strength of partial disclinations.

process. The broken line shows the ideal geometrical relationship between $\gamma$ and $\theta$. The observed angle $\theta$ during SPD continuously increases with $\gamma$ with a small deviation from the ideal relationship. The deviation could be attributed to the usage of the sine function to introduce the local shear strain. Despite the continuous increase in $\theta$ with $\gamma$, the critical value of $\gamma$ for grain subdivision during SPD is 0.7 . The physical meaning of the critical value $\gamma_{\mathrm{c}}$ is discussed in the next subsections.

\subsection{Mobility of partial disclinations}

The terminated band with misorientation is modeled as a disclination dipole, as shown on the left side of the inset of Fig. 7. The motion of the dipole parallel to the partial-disclination boundaries is known to increase the band length and contribute to the formation of misorientation and kink bands ${ }^{19)}$, which are closely related to grain subdivision. However, the observed motion of partial disclinations during SPD in this study is different from the above description; in our simulations, the partial disclinations move perpendicular to their boundaries along the specimen and follow the movement of the shear-strain zone caused by the DC layer. The partial disclinations travel along the specimen without producing grain subdivision when the shear strain is small, and the partial disclinations are left behind in the specimen when the strain is relatively large. In the latter case, deformation microstructures are observed in the specimens after SPD. To summarize briefly, the grain subdivision observed in the present simulations requires partial disclinations to be left inside of the specimen. Thus, partial disclinations with low mobility are important for the grain subdivision. The low mobility of the partial disclinations thought to be due to the large shear strain. Partial disclinations are accompanied by terminated boundaries with misorientations; hence, the mobility of partial disclinations is strongly related to the mobility of grain boundaries. To reveal the mechanism of the shear-strain dependence of the mobility of partial disclinations, we perform shear deformation tests on bicrystal models containing grain boundaries with various misorientation angles. Note that we do not consider the effect of the intrinsic stress fields due to the Frank vector $\omega$ of the partial disclination on the mobility of ones.

Figures $8(a)-8(d)$ show the structures of grain boundaries 


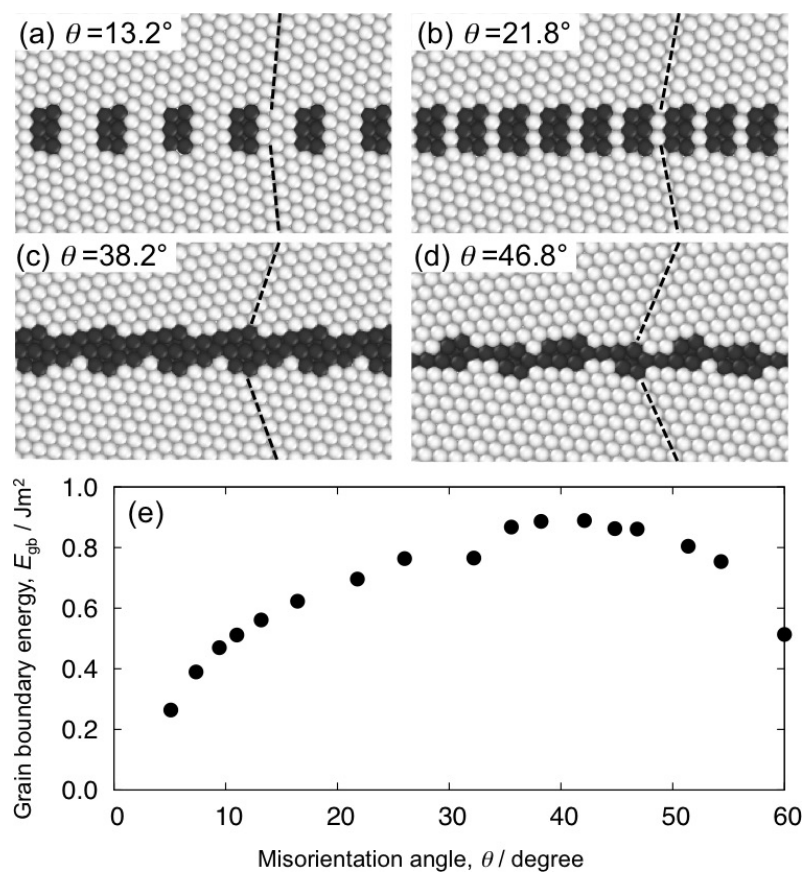

Fig. 8 (a-d) Grain boundary structures with various $\theta$ and (e) the relationship between the grain boundary energy and $\theta$.

with various misorientation angles $\theta$. Figure 8(e) shows the relationship between grain boundary energy $E_{\mathrm{gb}}$ and $\theta$. As shown in Fig. 8(e), an energy cusp is observed at $\theta=32^{\circ} . E_{\mathrm{gb}}$ increases with $\theta$ until $32^{\circ}$, and the grain boundary structures can be expressed by the edge dislocation arrays, as shown in Figs. 8(a) and 8(b). However, when $\theta$ exceeds $32^{\circ}, E_{\text {gb }}$ does not increase monotonically with $\theta$, and the grain boundary structures cannot be expressed by the edge dislocation array; instead, they can be expressed by the connection of structural units. Thus, the grain boundary structures transform from edge dislocation arrays to plane defects at the energy cusp at $\theta=32^{\circ}$.

Next, we investigate the grain boundary mobility by performing shear deformation tests on six bicrystal models with grain boundaries of $\theta=13.2^{\circ}, 21.8^{\circ}, 32.2^{\circ}, 38.2^{\circ}, 42.1^{\circ}$, and $46.8^{\circ}$. The analysis model is shown in the inset of Fig. 9(e). Each grain boundary is arranged at the center of the analysis model in the initial state. The dimensions of the analysis model are approximately $40 \mathrm{~nm} \times 1.7 \mathrm{~nm} \times 50 \mathrm{~nm}$, and periodic boundary conditions are adopted in the $x$ and $y$ directions. The dark-colored regions at the top and bottom of the analysis model represent DC layers. To make the grain boundaries migrate, the top DC layer is moved to the left at a velocity $v_{\|}$of $20 \mathrm{~m} / \mathrm{s}$, while the bottom layer is fixed. The grain boundary migration velocity $v_{\mathrm{m}}$ is measured for each $\theta$. Note that the GNBs interact with a large number of lattice dislocations during GNB migration (Fig. 6); hence, we also investigate the effect of intragranular lattice dislocations on $v_{\mathrm{m}}$ by introducing three edge dislocation dipoles in a grain where grain boundary migration occurs. The dislocation density is approximately $3 \times 10^{15} 1 / \mathrm{m}^{2}$, which is about ten times larger than the experimentally observed dislocation density of pure copper after $\mathrm{SPD}^{22)}$. The analysis temperature is kept at $300 \mathrm{~K}$ using the velocity scaling method.

Figures 9(a) and 9(c) show snapshots of grain boundary
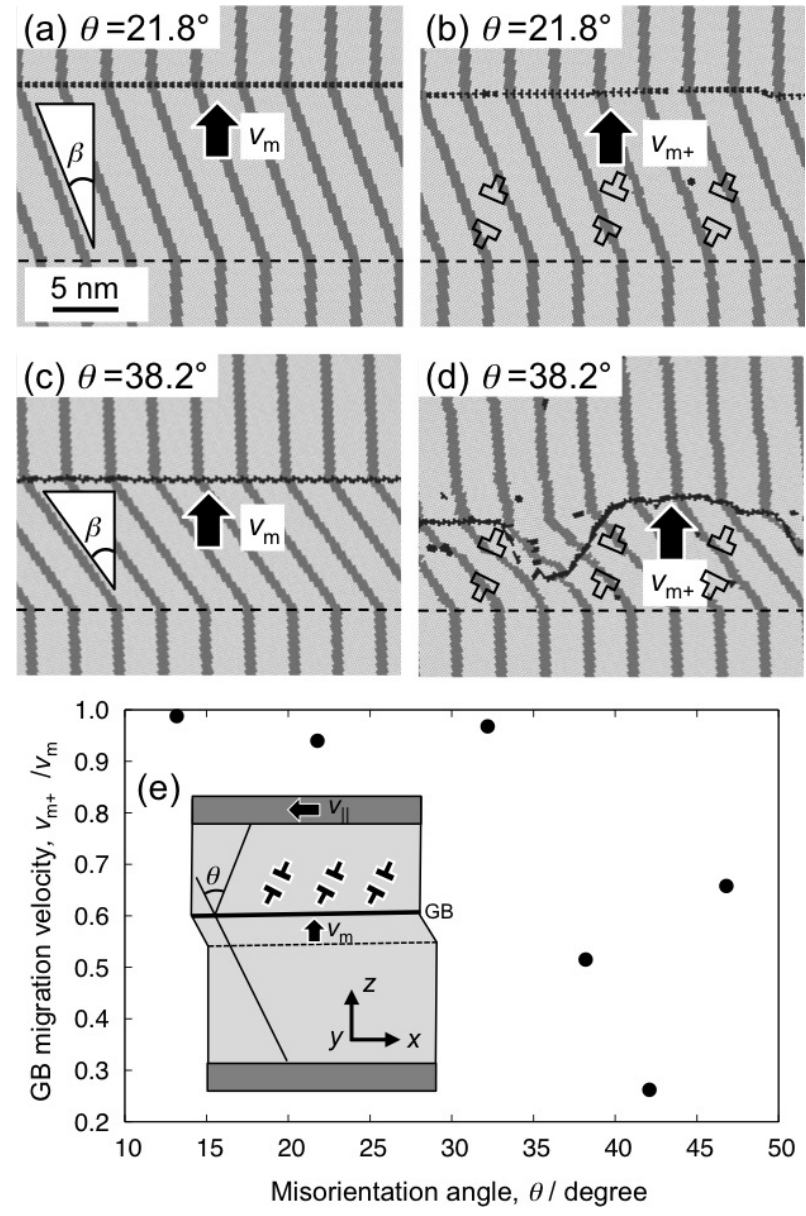

Fig. 9 Coupling motion of grain boundaries (a) with $\theta=21.8^{\circ}$, (b) with $\theta=$ $21.8^{\circ}$ interacting with intragranular lattice dislocations, (c) with $\theta=38.2^{\circ}$ and (d) with $\theta=38.2^{\circ}$ interacting with intragranular lattice dislocations. The initial positions of grain boundaries are show by horizontal broken lines. (e) Normalized migration velocity of grain boundaries interacting with intragranular lattice dislocations. Inserted figure represents the analysis model to the grain boundary coupling motion.

migration for $\theta=21.8^{\circ}$ and $38.2^{\circ}$ without intragranular lattice dislocations and simulation times of 0.3 and $0.45 \mathrm{~ns}$, respectively. To better understand the change in crystal orientation, the initial configuration of each model is marked by a gray-colored striped pattern perpendicular to the grain boundary in Figs. 9(a) and 9(c). Grain boundary migration toward the upper direction at velocity $v_{\mathrm{m}}$ is confirmed for both grain boundaries during shear deformation at velocity $v_{\|}$. After grain boundary migration, the stripe pattern is inclined indicating that the grain boundary migration yields plastic shear strain. The ideal coupling relation without grain boundary sliding is expressed as $v_{\|}=\beta v_{\mathrm{m}}$, where $\beta$ is the coupling factor that depends on the crystallographic parameters of the grain boundary $^{23)}$. The observed values of $\beta$ for all bicrystal models correspond well to the ideal coupling factor calculated by $2 \tan (\theta / 2)$ within an error of approximately $\pm 5 \%$; thus, the perfect coupling grain-boundary motion to shear deformation occurs to all grain boundaries. This result implies that partial disclinations can follow the movement of strain-gradient regions during SPD if the partial-disclination boundaries do the perfect coupling grain-boundary motion to shear deformation.

However, when partial-disclination boundaries interact 
with intragranular lattice dislocations, they cannot follow the movement of strain-gradient regions. Figures 9(b) and 9(d) show snapshots of grain boundary motion with $\theta=21.8^{\circ}$ and $38.2^{\circ}$ in the presence of intragranular lattice dislocations, and simulation times of 0.3 and $0.45 \mathrm{~ns}$, respectively. In the case of $\theta=21.8^{\circ}$, the boundary structure can be expressed by the edge dislocation array, as shown in Fig. 8(b), and the interaction with intragranular lattice dislocations has a negligible impact on the grain boundary migration velocity $v_{\mathrm{m}+}$ [Fig. 9(b)]. This is due to the indirect (elastic) interactions among grain boundary dislocations. Even if a grain boundary dislocation loses its mobility by interacting with an intragranular lattice dislocation, other grain boundary dislocations can still move without losing their mobility. On the other hand, for $\theta=38.2^{\circ}$, the boundary structure can be expressed by the connection of structural units, as shown in Fig. 8(c), and the intragranular lattice dislocations have a strong impact on the grain boundary motion [Fig. 9(d)]. This result can be attributed to the direct interactions among the structural units composing the large-angle grain boundaries. The grain boundary coupling motion requires collective structural change. When structural units in a local region interact with an intragranular lattice dislocation, the collective motion around the region becomes difficult because of the destruction of the periodicity of structural units. Consequently, the grain boundary mobility in the local region interacting with intragranular lattice dislocations decreases, and the grain boundary plane takes on a wavy shape, as shown in Fig. 9(d).

Figure 9(e) shows the dependence of $v_{\mathrm{m}+}$ normalized by $v_{\mathrm{m}}$ on $\theta$. Here, $v_{\mathrm{m}+}$ is the velocity of the center of gravity of a wavy grain boundary in the $z$ direction. The grain boundary mobility is clearly not influenced by interactions with intragranular lattice dislocations when the grain boundary structure is simply expressed by an edge dislocation array; however, the mobility is strongly affected by the intragranular lattice dislocations when the grain boundary structure can be expressed by the connection of structural units. Consequently, the mobility of partial disclinations, the boundaries of which interact with intragranular lattice dislocations, strongly depends on the structure of the partial-disclination boundaries, and the decrease in mobility causes the grain subdivision during SPD.

\subsection{A grain subdivision mechanism related to partial disclinations}

Here, we describe a mechanism for grain subdivision during SPD based on our simulation results. A large number of lattice dislocations are introduced by the large plastic strain during SPD. Partial disclinations are formed as GNBs by the rearrangement of lattice dislocations in strain-gradient regions (Fig. 6), and the misorientation angles of partial-disclination boundaries increase with local shear strain $\gamma$ (Fig. 7). The mobility of the partial disclinations, the boundaries of which interact with intragranular lattice dislocations, is very important for grain subdivision because the strain-gradient regions propagate during SPD. For partial-disclination boundaries with small-misorientation angles, the boundary structures are expressed by edge dislocation arrays, and the mobility of partial disclinations is not influenced by lattice dislocations. However, for partial-disclination boundaries with large-misorientation angles, the boundary structures are expressed by structural units, and the interaction with lattice dislocations decreases the mobility of the partial disclinations. Consequently, deformation microstructures with misorientation angles are formed by the residual partial disclinations after the SPD process, finally resulting in grain subdivision.

The observed misorientation angle of partial-disclination boundaries during the SPD simulation at $\gamma_{\mathrm{c}}$ (approximately $27^{\circ}$, as shown in Fig. 6) is in good agreement with the misorientation angle at which the mobility of symmetrical grain boundaries decreases $\left(\sim 32^{\circ}\right.$, as shown in Fig. 9). The small deviation between these two angles is due to the influence of the asymmetrical components ${ }^{24}$ of partial-disclination boundaries in strain-gradient regions and the intrinsic stress fields of partial disclinations on the coupling motion along with the different deformation conditions between the SPD process and the shear deformation test. Consequently, the critical $\gamma_{\mathrm{c}}$ required for grain subdivision could be determined by the misorientation angle of the partial-disclination boundaries at which the boundary structure transforms [Fig. 8(e)].

Our grain subdivision mechanism is also supported by some experimental results. Nakashima et al. reported the channel-angle dependence of grain size for an ECAP process $^{25)}$. The channel angle of the ECAP process corresponds well to the local shear strain $\gamma$ observed in the present simulations. As the channel angle nears $90^{\circ}$, the angle at which specimens deform at right angles, grain refinement occurs efficiently. Because strain gradients increase in specimens fabricated via ECAP with the channel angle close to $90^{\circ}$, strain gradients also play an important role in ECAP grain refinement. For the HPT process, Todaka et al. reported the effect of strain gradient on hardness; the hardness increases from the center of the specimen to the outside ${ }^{26)}$. Therefore, the microstructures of the specimens can also be influenced by the strain gradients induced by the HTP process. Although the present simulations of shear-strain deformation of nanoscale specimens cannot capture all the phenomena that occur in an actual SPD process, the obtained results qualitatively reflect the process of grain refinement during real SPD.

As discussed above, the atomic simulations reported herein reveal a new mechanism of grain subdivision related to the mobility of the partial disclinations induced by strain gradients during SPD. To confirm the generality of the proposed mechanism, we performed the same SPD simulations using the Morse potential, a two-body potential, for a simple two-dimensional triangle-lattice model with three equivalent slip systems. The obtained results were the qualitatively the same as those obtained using the EAM potential, although critical values $\gamma_{\mathrm{c}}$ differed due to different defect properties (e.g., energy and structure) between the interatomic potentials. Thus, these results support the generality of the proposed mechanism. In addition to previous research on the relationship between grain subdivision and partial disclinations using the evolution equation ${ }^{27)}$ and an energetic approach ${ }^{28)}$, both of which consider the intrinsic stress fields of the partial disclinations, our atomic-simulation results help provide a more complete of atomic-level grain subdivision and shed light on the mechanism of grain refinement during SPD. 


\section{Conclusion}

In this study, we detailed a mechanism of grain subdivision based on critical shear strain related to partial disclinations using atomic simulations. In the simulations, specimens were locally deformed by simple shear at different shear strain magnitudes (0.2-1.2) imposed by the outside frame, and the local regions of shear deformation propagated along the specimens. Partial disclinations formed to accommodate the strain gradients, which increased with the magnitude of shear strain. When the shear strain magnitude exceeded a critical value, deformation microstructures are formed (i.e., grain subdivision occurred), by leaving the partial disclinations inside the specimens after the deformation process. This critical value of shear strain can be explained by the reduction in the mobility of the partial disclinations due to the fundamental structural transition of their boundaries from an array of lattice dislocations to a sequence of structural units. These results will shed light on the mechanism of grain refinement by SPD processes.

\section{Acknowledgments}

The authors acknowledge financial support from MEXT KAKENHI Grant numbers 22102002 and 22102007 (Grantsin-Aid for Scientific Research on Innovative Areas, "Bulk Nanostructured Metals").

\section{REFERENCES}

1) B. S. Altan, I. Miskioglu, G. Purcek, R.R. Mulyukov, R. Artan: Severe plastic deformation: towards bulk production of nanostructured materials, NOVA Science Publishers, New York (2006).

2) R.Z. Valiev and T.G. Langdon: Prog. Mater. Sci. 51 (2006) 881-981.

3) N. Hansen: Metallurgical and Materials Transactions A, 32A (2001), 2917-2935.

4) X. Huang, N. Tsuji, N. Hansen and Y. Minamino: Mater. Sci. Eng. A 340 (2003) 265-271.

5) A. Sutton and R. Balluffi: Interfaces in Crystalline Materials, Oxford
Science, New York (1995).

6) If the misorientation angle of GNBs has twist components, the structure of the GNBs can be expressed by the two or more types of screw dislocations.

7) M.F. Ashby: Philos. Mag. 21 (1970) 399-424.

8) R.Z. Valiev, Y.V. Ivanisenko, E.F. Rauch and B. Baudelet: Acta Mater. 44 (1996) 4705-4712.

9) Y. Iwahashi, J. Wang, Z. Horita, M. Nemoto and T.G. Langdon: Scr. Mater. 35 (1996) 143-146.

10) R.Z. Valiev and T.G. Langdon: Prog. Mater. Sci. 51 (2006) 881-981.

11) Y. Saito, H. Utsunomiya, N. Tsuji and T. Sakai: Acta Mater. 47 (1999) 579-583.

12) A. E. Romanov and V. I. Vladimirov; Disclinations in crystalline solids, in Dislocations in Solids, ed. F. R. N. Nabarro. Vol. 9, North Holland, Amsterdam (1992).

13) K. Nakashima, Z. Horita, M. Nemoto and T.G. Langdon: Mater. Sci. Eng. A 281 (2000) 82-87.

14) G.I. Raab: Mater. Sci. Eng. A 410-411 (2005) 230-233.

15) Y. Mishin, M. J. Mehl, D. A. Papaconstantopoulos, A. F. Voter and J. D. Kress: Phys. Rev. B 63 (2001) 224106(1-16).

16) M. P. Allen and D. J. Tildesley: Computer Simulation of Liquids, Oxford University Press, New York (1987).

17) J. Dana Honeycutt and H.C. Andersen: J. Phys. Chem. 91 (1987) 49504963.

18) M. Murayama, J.M. Howe, H. Kidaka and S. Takaki: Science 295 (2002) 2433-2435

19) A.E. Romanov and A.L. Kolesnikova: Prog. Mater. Sci. 54 (2009) 740769.

20) T. Shimokawa, M. Tanaka, K. Kinoshita, and K. Higashida: Phys. Rev. B 83 (2011) 214113(1-13).

21) I.A. Ovid'ko: Phil. Trans. R. Soc. A 373 (2015) 20140129.

22) Y. Miyajima, S. Okubo, H. Abe, H. Okumura, T. Fujii, S. Onaka and M. Kato: Mater. Charact. 104 (2015) 101-106.

23) J.W. Cahn, Y. Mishin and A. Suzuki: Acta Mater. 54 (2006) 4953-4975.

24) Z.T. Trautt, A. Adland, A. Karma and Y. Mishin: Acta Mater. 60 (2012) 6528-6546.

25) K. Nakashima, Z. Horita, M. Nemoto and T.G. Langdon: Acta Mater. 46 (1998) 1589-1599.

26) Y. Todaka, M. Umemoto, J. Yin, Z. Liu and K. Tsuchiya: Mater. Sci. Eng. A 462 (2007) 264-268.

27) M. Seefeldt, L. Delannay, B. Peeters, E. Aernoudt and P. Van Houtee: Acta Mater. 49 (2001) 2129-2143.

28) T.S. Orlova, A.A. Nazarov, N.A. Enikeev, I.V. Alexandrov, R.Z. Valiev and A.E. Romanov: Phys. Solid State 47 (2005) 845-851. 